\title{
In-line Phase Contrast Tomography of the Breast with a Dedicated Micro-CT Scanner
}

\author{
Giovanni Mettivier, Kristina Bliznakova, Antonio Sarno, Francesca Di Lillo, Roberta Castriconi, \\ Paolo Russo
}

\begin{abstract}
We performed laboratory investigations of the image quality for in-line phase contrast micro tomography dedicated to the breast, using a 3D breast phantom. We employed the cone-beam, microfocus (7-micrometer focal spot size) computed tomography (CT) scanner developed in-house for investigation of high resolution, absorption based, and phase contrast based, $\mathrm{X}$-ray imaging of the breast for cancer diagnosis, at dose levels comparable to two-view mammography. CT slices were reconstructed (FDK algorithm) from phase contrast projection images as well as from phase retrieved projections (ANKAphase algorithm), under the assumption of spatially uniform distribution in the object volume of the ratio of the $\mathrm{X}$-ray optical coefficients of the sample material, at the effective energy of the X-ray beam $(80 \mathrm{kV}, \mathrm{HVL}=6.11 \mathrm{~mm} \mathrm{Al})$. Microcalcifications were best visible after phase retrieval, down to $0.23 \mathrm{~mm}$ size.
\end{abstract}

\section{INTRODUCTION}

$P^{\prime}$ HASE CONTRAST imaging dedicated to the breast has been the subject of extensive research by various groups in recent years [1]. Adopting phase contrast detection, 2D and $3 \mathrm{D}$ techniques as mammography, breast tomosynthesis as well breast CT have been investigated. Most research is focused on the use of grating-based techniques, including phase contrast interferometry and phase contrast differential imaging, but which technique offers the best tradeoff for clinical implementation, is still matter of investigation.

We have developed a cone beam CT scanner dedicated to the breast $[2,3]$, which employs a microfocus X-ray tube and a high resolution detector: it permits to exploit, at least to some extent, the presence of in-line phase contrast effects in the beam propagation from the source to the detector through the object, due to the small size of the X-ray source. Moreover, it is expected to provide suitable high-spatial resolution for improved visibility of microcalcifications.

In this work, we investigate the performance of this setup for in-line phase contrast micro-CT dedicated to the breast, in phantom tests. A phase retrieval algorithm applied to projections before FDK CT reconstruction produced both phase contrast CT and phase CT images of the breast phantoms, for visibility tests on masses and microcalcifications

Manuscript received October 17, 2016. G.M., A.S., F.D.L., R.C. and P.R. are with Universita' di Napoli Federico II, Dipartimento di Fisica "Ettore Pancini", and also with INFN Sezione di Napoli, I-80126 Napoli, Italy, (telephone: +39-081-676339, e-mail: mettivier@na.infn.it). K.B. is with Department of Software and Internet Technology, Technical University of Varna, Varna, Bulgaria.

\section{MAterials AND Methods}

The laboratory setup for phase contrast cone-beam microCT dedicated to the breast (Fig. 1) consisted of a microfocus X-ray tube, a high resolution CMOS flat panel detector with $50-\mu \mathrm{m}$ square pixels and $12 \times 12 \mathrm{~cm}^{2}$ sensitive area (Hamamatsu mod.C4791CA-02) and a computer controlled rotating gantry with variable magnification [3]. The X-ray tube (Hamamatsu mod. L8121-03) was operated at a constant tube voltage of $80 \mathrm{kV}$ and at a tube current of $0.125 \mathrm{~mA}$ and an exposure time of $106 \mathrm{~s}$. The focal spot size was $7 \mu \mathrm{m}$. The source-to-object distance was $\mathrm{R}_{1}=576 \mathrm{~mm}$, the object-to-detector distance was $\mathrm{R}_{2}=614 \mathrm{~mm}$. The added filtration was $0.3 \mathrm{~mm} \mathrm{Cu}$ and the HVL was $6.11 \mathrm{~mm} \mathrm{Al}$.

The breast phantom (BR3D mod. 020, CIRS Inc., Norfolk, VA, USA) (Fig. 2b) consisted of 5 slabs, each 1-cm thick, of BR50/50 material simulating 50\% glandular breast, with two components mixed to simulate locally heterogeneous glandular and adipose tissue distributions; one of the slabs contains mass, fibers and microcalcification details. Another test object (CIRS mod. 014AD, BR50/50 material, 6 slabs for 8.5-cm thickness, containing a slab with details) (Fig. 2a) was also used, for planar imaging tests of spatial resolution and beam hardening evaluations. Projections were acquired in magnification (phase contrast condition) and then either processed directly with the CT reconstruction algorithm for cone beam (FDK) filtered backprojection ("phase contrast $\mathrm{CT}^{\prime \prime}$ ), or passed to a phase retrieval algorithm (ANKAphase) [4] based on the Paganin's filter [5] and then to the FDK algorithm ("phase retrieval CT").

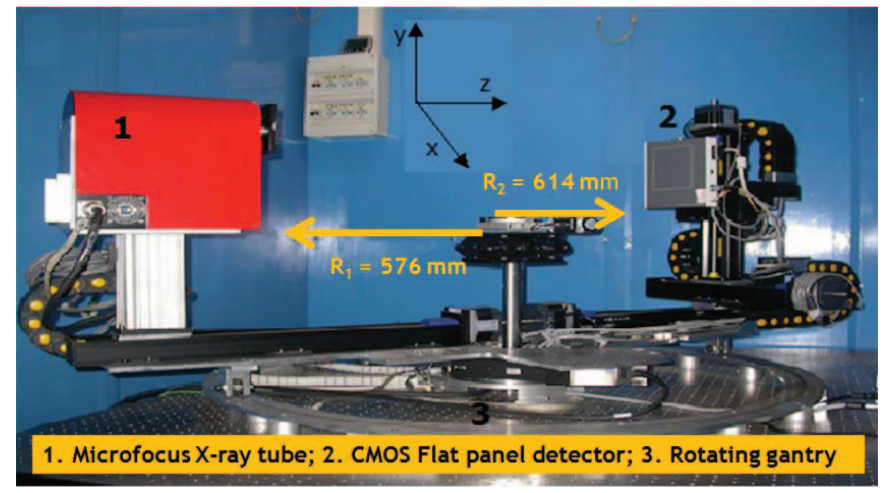

Fig. 1. Photo of the experimental setup. 1) Microfocus X-ray tube; 2) CMOS Flat panel detector; 3 ) rotating gantry. 

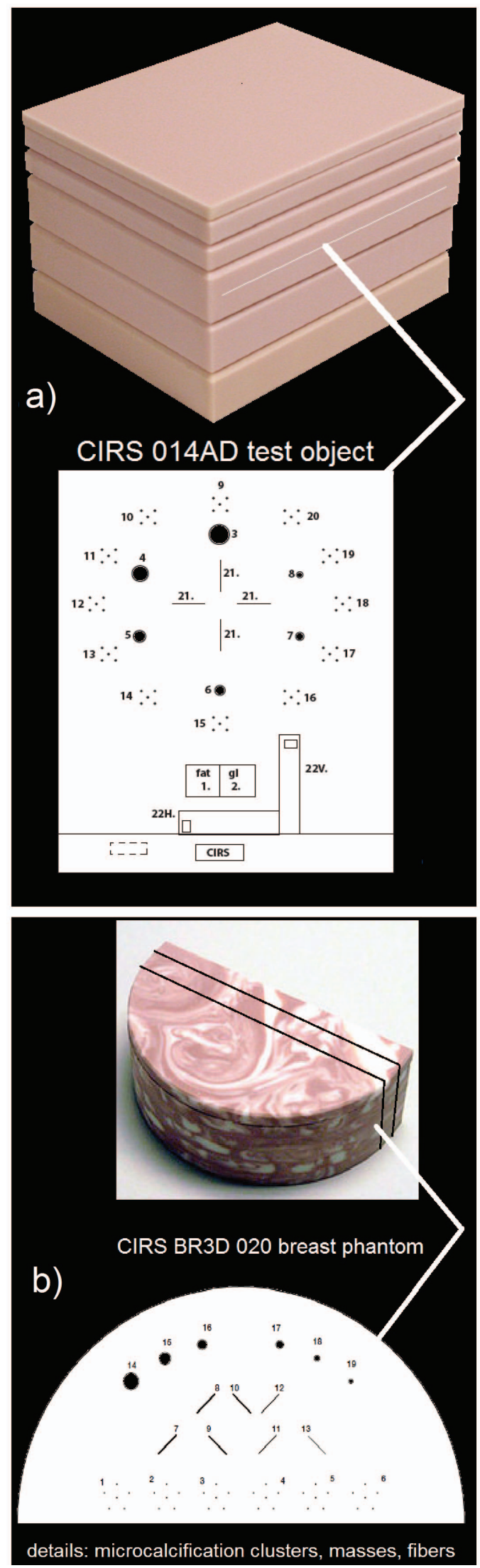

Fig. 2. a) Homogeneous BR50/50 test object, $8.5-\mathrm{cm}$ thick, including a slab with details, for 2D imaging; b) two-components BR50/50 breast phantom, composed of five slabs each 1-cm thick; the central slab embeds details for 3D imaging tests: the region indicated by the black lines is the ROI selected for details analysis in figs. 5 and 6.
The phase retrieval algorithm assumes that the sample is made of a material with spatial uniform distribution of the ratio $\delta / \beta$, where $n(E)=1-\delta(E)+i \beta(E)$ is the complex refractive index of the sample material at the effective energy $E$ of the X-ray photon spectrum, $\delta$ is the decrement of the real part of $n$ (responsible for the phase shift introduced by the sample) and $\beta$ is the absorption index (proportional to the linear attenuation coefficient of the material at energy $E$ ). Under this assumption, for a locally heterogeneous material (e.g. as for the breast phantom BR3D) any contrast shown in the phase map comes from the local difference between the assumed $\delta / \beta$ value (calculated with the code XOP v2.3 at the effective energy of the X-ray beam) [6] and its actual value at the given position in the map.

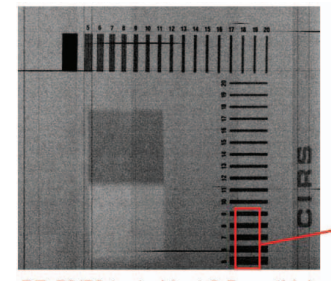

a)

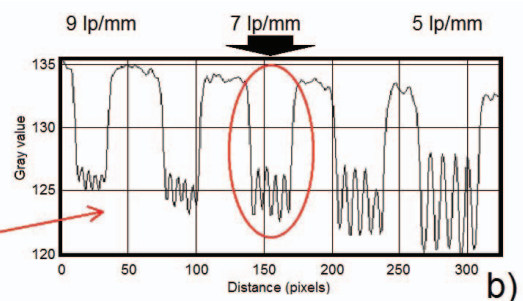

Air Kerma 1 mGy; MGD $\sim 0.45$ mGy

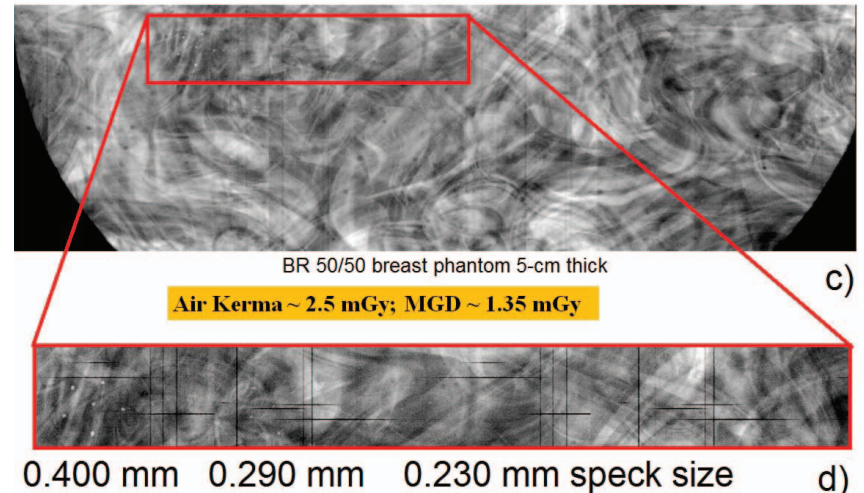

d)

Fig. 3. a) Phase contrast projection radiography of the CIRS mod. 014AD test object, b) line profile along the resolution bar pattern showing that the spatial resolution reaches $7 \mathrm{lp} / \mathrm{mm}$. c) Phase contrast projection radiography of the CIRS BR3D breast phantom. Image magnification $\cong 2$. d) Zoomed image of three microcalcification clusters of the BR3D phantom.

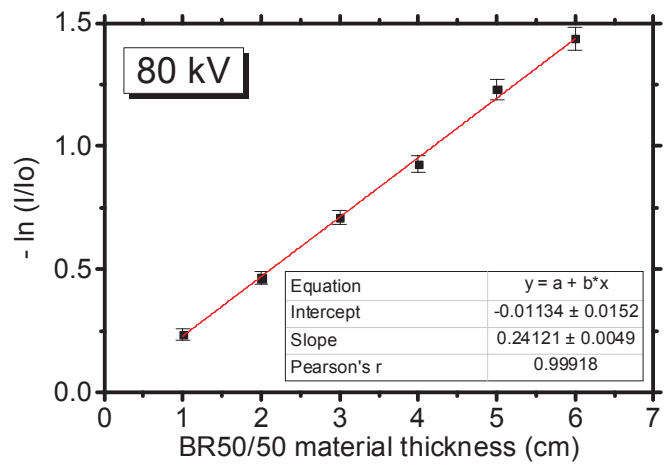

Fig. 4. Logarithmic attenuation measured with the flat panel detector with increasing thicknesses of BR50/50 material, showing a linear trend (continuous fitting line) which indicates limited influence of the beam hardening effect up to 6-cm material thickness. The slope of the linear fit indicates an effective attenuation coefficient of $0.241 \mathrm{~cm}^{-1}(42.5 \mathrm{keV}$ beam effective energy). 


\section{RESUlTS}

Figures 3a,b show a planar imaging test with the BR50/50 test object, which indicates that the system spatial resolution in $2 \mathrm{D}$ imaging is as high as $7 \mathrm{lp} / \mathrm{mm}$. When the twocomponents breast phantom is imaged in a craniocaudal view (Fig. 3c), the effects of using a microfocus source and a magnification $\cong 2$ are visible as an improved visualization of border regions between adipose and fibroglandular simulated materials, with respect to contact radiography. Microcalcification clusters down to $0.230-\mathrm{mm}$ size can be detected (Fig. 3c). The average pixel value is exponentially dependent on the thickness of the BR50/50 material (Fig. 4), indicating a weak dependence on the beam hardening effect in this thickness range we note that (the slope of this graph shows an attenuation coefficient of $0.241 \mathrm{~cm}^{-1}$ and a corresponding beam effective energy of $42 \mathrm{keV}$ ).

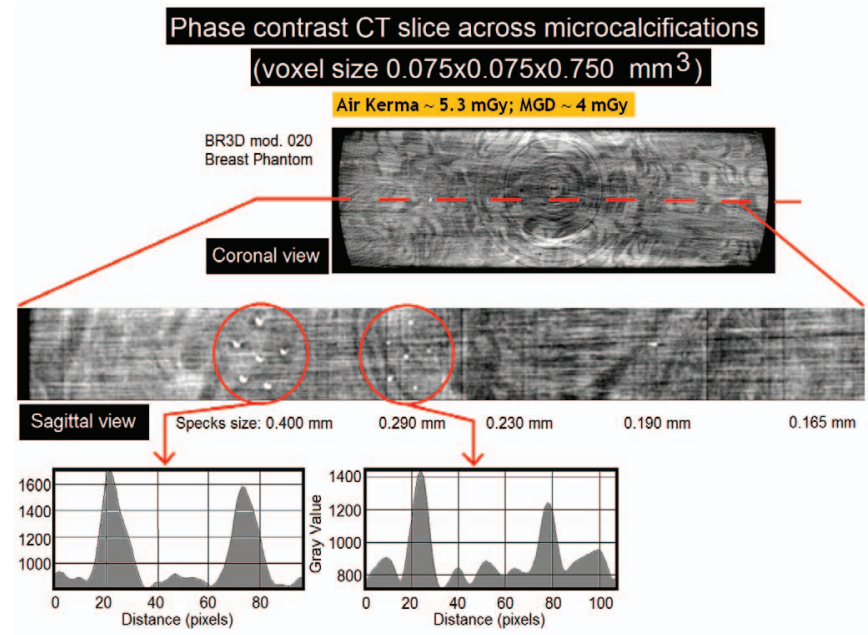

Fig. 5. Phase contrast CT slice of the 3D breast phantom, for microcalcification visibility test. Specks down to $0.290 \mathrm{~mm}$ are clearly visible; the bottom graphs show line profiles across two $\mathrm{CaCO}_{3}$ specks in the clusters. FDK reconstruction, 360 views.

Figure 5 shows the phase contrast CT (a single coronal slice and corresponding sagittal view) of the CIRS BR3D phantom, where microcalcification clusters of 0.400 and $0.290 \mathrm{~mm}$ are clearly visible. With respect to these CT data, phase retrieval (Fig. 6) permits a better visibility of microcalcifications (from $0.400 \mathrm{~mm}$ to $0.230 \mathrm{~mm}$ ), due to a higher contrast, at the same level of mean glandular dose (estimated MGD $=4 \mathrm{mGy}$ for the 50/50 breast phantom) comparable to that of two-view mammography for the same compressed breast.

The present findings show that the use of a microfocus Xray tube in a clinically-compatible $\mathrm{CT}$ setup dedicated to the breast produces some degree of phase-contrast effects in propagation based imaging, which can be exploited for an improvement in visibility of masses and microcalcifications, at mean glandular dose levels comparable to that of two-view mammography. A key feature of our apparatus, relying on a propagation based phase contrast technique, is its high spatial resolution $\left(3.8 \mathrm{~mm}^{-1}\right.$ in the radial direction at $\left.10 \% \mathrm{MTF}\right)$ [7]. Other approaches for breast imaging use X-ray CT interferometric techniques employing more complex setups [8], or synchrotron radiation CT at large facilities $[9,10]$.

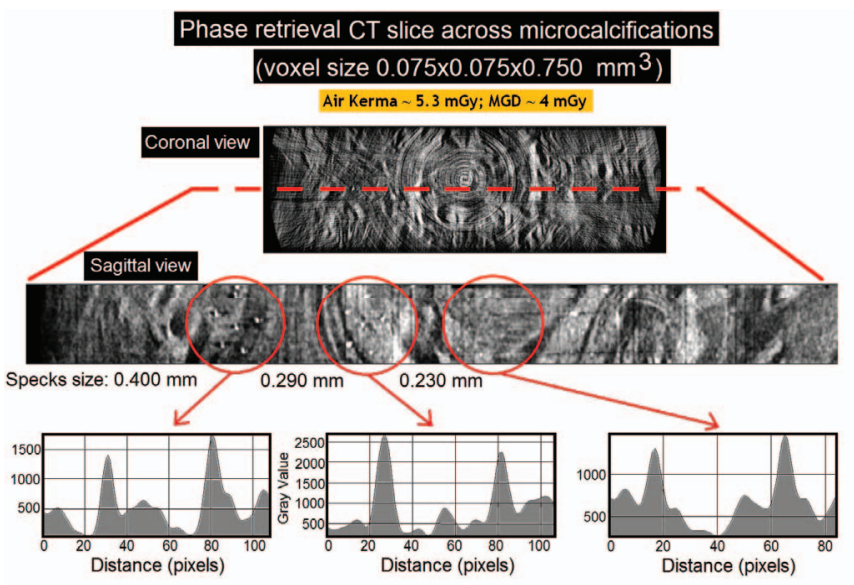

Fig. 6. Phase retrieval CT slice (from the same projections used for fig. 5) of the 3D breast phantom; specks down to $0.230 \mathrm{~mm}$ can be observed.

\section{ACKNOWLEDGMENTS}

This work is supported by MaXIMA project: Three dimensional breast cancer models for $\mathrm{x}$-ray imaging research. This project has received funding from the European Union's Horizon 2020 research and innovation programme under grant agreement No 692097.

\section{REFERENCES}

[1] S. D. Auweter, et al., "X-ray phase-contrast imaging of the breastadvances towards clinical implementation," Br. J. Radiol., vol. 87, no. 1034, pp. 1-8, Jan. 2014.

[2] A. Sarno, G. Mettivier, P. Russo, "Dedicated breast computed tomography: basic aspects," Med. Phys., vol. 42, no. 6, pp. 2786-2804, June 2015

[3] G. Mettivier et al., "Dedicated scanner for laboratory investigations on cone-beam CT/SPECT imaging of the breast," Nucl. Instr. Meth. A, vol. 629 (1), pp. 350-356, 2011.

[4] T. Weitkamp, D. Haas, D. Wegrzynek, and A. Rack, "ANKAphase: software for single-distance phase retrieval from inline x-ray phasecontrast radiographs," J. Synchrotron Radiat., vol. 18, pp. 617-629, 2011.

[5] D. Paganin et al., "Simultaneous phase and amplitude extraction from a single defocused image of a homogeneous object," J. Microsc., vol. 206, pp. 33-40, 2006.

[6] M. Sanchez del Rio and R. J. Dejus XOP (X-Ray Oriented Programs). European Synchrotron Radiation Facility (ESRF), 2003.

[7] A. Sarno, G. Mettivier, F. Di Lillo, M. Cesarelli, P. Bifulco, and P. Russo, "Cone-beam micro computed tomography dedicated to the breast," Med. Phys. Eng., 2016 (in press).

[8] A. Bravin, P. Coan, and P. Suortti, "X-ray phase-contrast imaging: from pre-clinical applications towards clinics," Phys. Med. Biol. vol. 58, R1-R35, 2013.

[9] R. Longo, F. Arfelli, R. Bellazzini, U. Bottigli, A. Brez, F. Brun et al., "Towards breast tomography with synchrotron radiation at Elettra: first images," Phys. Med. Biol. vol. 61, pp. 1634-1649, 2016.

[10] A. Sarno et al., "Imaging performance of phase-contrast breast computed tomography with synchrotron radiation and a CdTe photoncounting detector," Phys. Medica, vol. 32, pp. 681-690, 2016. 